\title{
Use of a prohibition order after a large outbreak of gastroenteritis caused by norovirus among function attendees
}

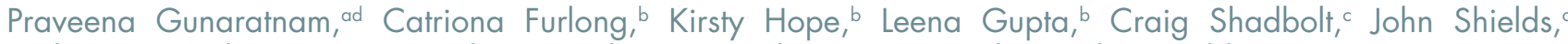 \\ Rodney McCarthy, ${ }^{\mathrm{c}}$ Rowena Boyd, ${ }^{\mathrm{b}}$ Essi Huhtinen, ${ }^{\mathrm{b}}$ Sophie Norton ${ }^{\mathrm{b}}$ and Siranda Torvaldsen ${ }^{\mathrm{d}}$ \\ Correspondences to Praveena Gunaratnam (e-mail: pguna@doh.health.nsw.gov.au)
}

Introduction: In May 2011, an outbreak of acute gastroenteritis occurred among guests attending two functions (Function A and B) at a local function centre in Sydney, Australia. The Sydney South West Public Health Unit and the New South Wales (NSW) Food Authority sought to determine the cause of the outbreak and implement control measures.

Methods: A retrospective cohort study was planned. A complete guest list was unavailable, so guests who could be contacted were asked to provide details of other guests. Attendee demographics, symptom profile and food histories were obtained using a standard response questionnaire. Stool samples were requested from symptomatic guests. The NSW Food Authority conducted a site inspection.

Results: Of those interviewed, $73 \%$ of Function A guests and $62 \%$ of Function B guests were ill, with mean incubation times of 27 and 23 hours respectively. Diarrhoea was the most common symptom. Three stool samples and four environmental swabs were positive for norovirus. One food handler reported feeling ill before and during the functions. A prohibition order was used to stop food handlers implicated in the outbreak from preparing food.

Discussion: This outbreak strongly suggests transmission of norovirus, possibly caused by an infected food handler. Regulatory measures such as prohibition orders can be effective in enforcing infection control standards and minimising ongoing public health risk.

$\mathrm{N}$ orovirus is well recognized as the leading cause of acute gastroenteritis worldwide, and in Australia alone there are an estimated 1.8 million cases every year. ${ }^{1}$ Outbreaks are most common in settings such as aged care facilities, hospitals and restaurants where people are in close proximity to one another. ${ }^{2}$ Food handlers have been identified as the route of transmission in numerous norovirus outbreaks, with uncooked foods, such as salads, most likely to be contaminated..$^{3-9}$ Infected people can continue to shed norovirus after cessation of symptoms, with one study finding that stool samples were positive by polymerase chain reaction (PCR) for norovirus up to three weeks after the onset of illness. ${ }^{10}$

In New South Wales (NSW), the responsibility for investigating suspected outbreaks of foodborne illness and implementing control measures is shared between the local public health unit, which conducts the epidemiological investigation, and the NSW Food Authority, which conducts the environmental investigations. Investigations are conducted in line with national and state guidelines. ${ }^{11,12}$

The NSW Food Act 2003 allows the NSW Food Authority to sanction establishments that are not complying with food safety standards. In more serious circumstances, where food safety standards are breached and the NSW Food Authority believes action is needed to prevent or mitigate risk to public health, a prohibition order may be served. ${ }^{13}$ Food legislation in most Australian jurisdictions contain similar provisions.

A prohibition order prevents the use of specific equipment and/or the sale of particular or all foods from a premise. A prohibition order remains in place until a Certificate of Clearance is issued by the NSW Food Authority stating that the business is fit

\footnotetext{
New South Wales Public Health Officer Training Program, New South Wales Health, Sydney, Australia.

Public Health Unit, Sydney South West Area Health Service, Sydney, Australia.

Foodborne Illness Investigation Unit, New South Wales Food Authority, Sydney, Australia.

School of Public Health and Community Medicine, University of New South Wales, Sydney, Australia.

Submitted: 27 January 2012; Published: 29 May 2012

doi: 10.5365/wpsar.2011.3.1.008
} 
to handle food. Conditions for issuing a Certificate of Clearance can include sufficient cleaning and disinfection of the premises, food safety training for staff and documentation confirming that food handlers employed at the premises are not having, or carrying, a foodborne disease. ${ }^{13}$

In May 2011, the NSW Food Authority notified Sydney South West Public Health Unit of three separate groups totalling 31 people who had become ill with gastrointestinal symptomsfollowing attendanceatadinner two days earlier at a local function centre (Function A). On that same day, a local Emergency Department reported two additional unwell people, unknown to each other, who reported attending Function A with family and friends. This paper describes the investigation of the outbreak and control measures taken to minimize ongoing public health risk.

\section{METHODS}

\section{Epidemiological Investigation}

A retrospective cohort study was planned to help determine the causative agent and its transmission route i.e. person-to-person or foodborne.

Preliminary information from the NSW Food Authority indicated a lunch was also held the following day at the same venue (Function B). Contact details for event organizers and a small number of the guests attending one or both functions were obtained through the NSW Food Authority. A complete guest list was unavailable from event organizers, only details of the person who purchased the tickets for their group. Guests interviewed were asked to provide names and contact details of other attendees, thus generating a list of 105/260 (40.3\%) guests attending Function A and 46/150 (30.6\%) attending Function B.

Demographics, symptom profile and detailed food histories were obtained using a standard initial response questionnaire. The food history section was updated to include: foods consumed at the function; illness or contact with ill people before the function; and to determine if ill persons were more likely to sit together, seating placement of persons within the function room.

The NSW Food Authority also provided contact details for six food handlers who had worked at the function centre over the two days. Staff demographics symptom profile, food histories and hours of work were obtained using a standardized questionnaire.

Interviews were administered by telephone by public health officers at Sydney South West Public Health Unit over the six days following notification. Where contact was not possible on the first phone call, the public health officer would attempt up to three repeat phone calls at different times and on different days to reach the guest.

A case was defined as a person who has vomiting or diarrhoea with nausea or abdominal pain and attended either Function A or B. A secondary case was defined as a contact of a confirmed case who developed vomiting or diarrhoea with nausea or abdominal pain more than 72 hours after the function occurred.

Statistical analysis of interview data was carried out using Statistical Analysis Software (SAS) version 9.2. The detailed cohort analysis is not presented due to low response rate.

\section{Environmental Investigation}

A food premises inspection was conducted by the NSW Food Authority the day following notification. Managers and food handlers were interviewed regarding infection control, preparation techniques and food storage. During the inspection the menu was reviewed and swabs were taken from around the premises. Samples were not available for all foods consumed, but residual samples of the octopus, black olives, feta cheese and tzatziki were taken to test for viral particles and bacterial contamination.

\section{Microbiological Investigation}

Stool samples were requested from symptomatic guests and food handlers. Specimens were tested for viruses (norovirus, rotavirus and adenovirus) by enzyme immunoassay (EIA) at two public hospital laboratories, using the RIDASCREEN norovirus EIA kit (R-Biopharm AG, Darmstadt, Germany), which tests for the norovirus genogroups I and II. Specimens were also tested for bacterial pathogens (Salmonella, Shigella and Campylobacter) using PCR or culture, for Clostridium difficile by EIA and for ova, cysts and parasites by microscopy. 
Norovirus genogrouping and phylogenetic analysis was conducted at the University of NSW using real-time reverse transcription PCR. This analysis compared two of the positive stool samples with the environmental norovirus isolates found at the premises. The RNA was extracted from the stool samples using the Viral RNA Mini kit (Qiagen Inc., Valencia, CA, USA) and DNA synthesized using the SuperScript VILO cDNA Synthesis kit (Invitrogen, Grand Island, NY, USA). RNA extraction and DNA synthesis from the environmental samples was done at the Molecular Microbiology Laboratory at the Division of Analytical Laboratories using an in-house kit for RNA extraction and the cDNA Synthesis kit (Bioline, Tauton, MA, USA).

\section{RESULTS}

\section{Epidemiological Investigation}

Of the 151 guests whose contact details were obtained, 109 (72\%) were interviewed. Of the 109, 88 attended Function A and 21 attended Function B. Two people interviewed attended both functions. Two people declined to be interviewed and the remaining 40 could not be contacted by phone because a wrong number was provided or there was no answer after repeated calls. Of the people interviewed, 64 (73\%) Function A guests and 13 (62\%) Function B guests were ill following their respective function.

Most cases interviewed were between the ages of 60 and 79. The median age of cases was 68 years (range 6 to 84). Diarrhoea was the most common symptom among cases (92\% at Function A, $77 \%$ at Function B), followed by nausea ( $84 \%$ and $69 \%$ ) and vomiting ( $84 \%$ and $69 \%$ ). Twenty-two (34\%) of the Function A cases and seven (54\%) of the Function B cases visited their doctor or an emergency department.

The time between attendance and onset of symptoms ranged from 7 to 53 hours (mean 27 hours) for Function $A$ cases and from 3 to 30 hours (mean 23 hours) for Function B cases. One guest had an incubation time of over four days and was classified as a secondary case. No guests reported developing symptoms at the function, though two guests attending Function A had short onset times of seven and eight hours after the event. One Function $B$ guest who also attended Function $A$ reported onset of symptoms three hours after Function B (Figure 1).

Figure 1. Onset of symptoms among cases in a gastroenteritis outbreak in Sydney, May 2011

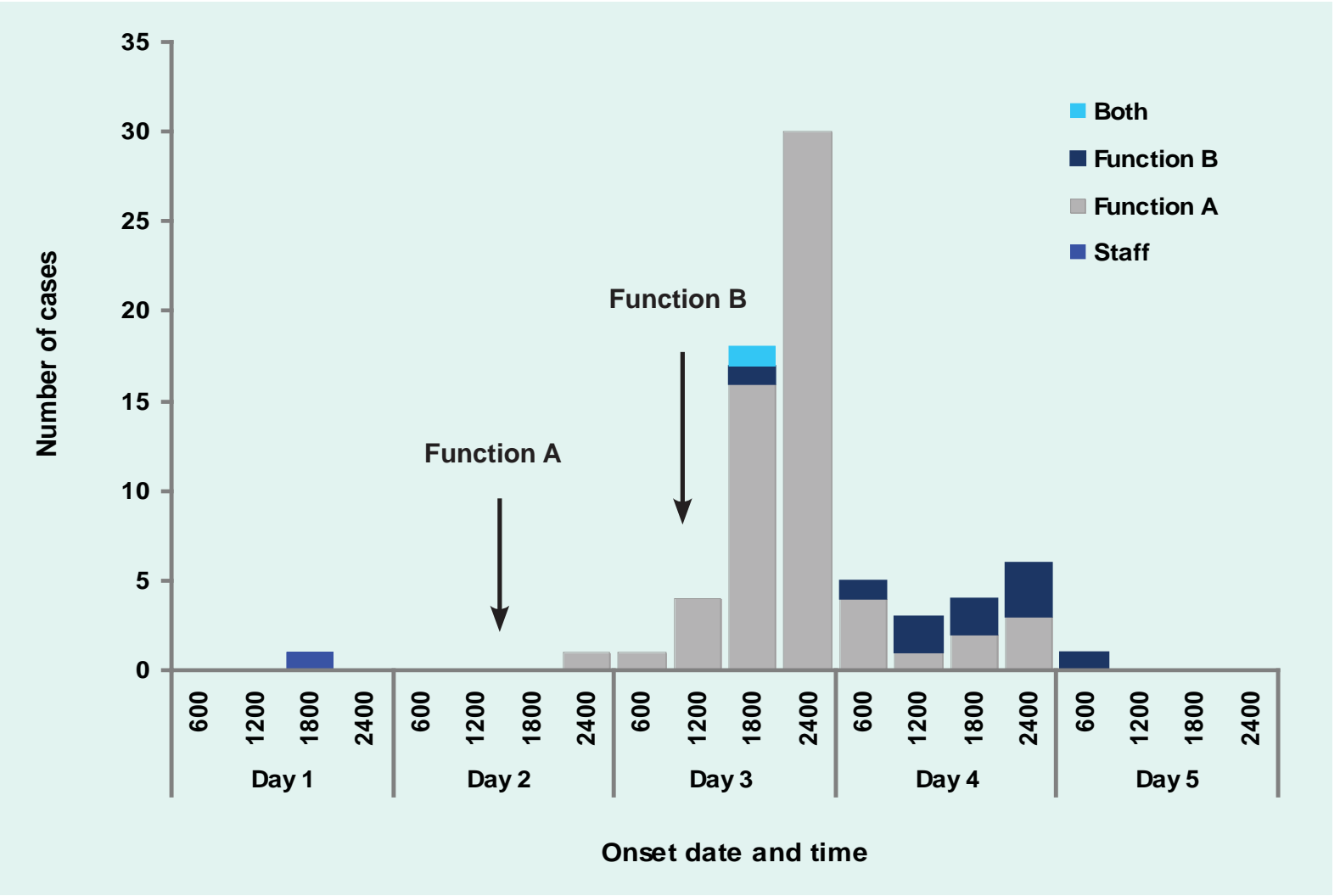


One food handler reported feeling ill 24 hours before the start of Function $A$, with diarrhoea and vomiting starting on the morning of Function $A$ and continuing for four days. Prior to Function $A$ the food handler vomited once at work, though precise location was not reported. The food handler prepared food for both functions while unwell. No other food handler reported illness. Information about staff food consumption and symptoms was not included in the analysis due to small numbers.

The mean duration of illness for Function $A$ cases was 48 hours (range 2-144 hours) with nine guests still unwell at the time of interview. The mean duration for Function B cases was also 48 hours (range 9-96 hours) with one guest still unwell at the time of interview.

Exposure information was collected for possible food exposures and person-to-person transmission, with the intention of calculating relative risks for each of the 20 foods available at either one or both functions. However, due to the low response rate, it was not possible to undertake valid analysis of the food exposure data. Information on seating arrangements revealed ill persons were no more likely to be seated in any particular area of the function room compared to those not reporting illness and was also not analysed further.

\section{Environmental Investigation}

\section{Food hygiene inspection}

Catering at both events consisted of mostly processed and fried foods. Items made on site included Greek salad and tzatziki. The ill food handler was involved in making the tzatziki and preparing and placing processed foods onto individual plates. Assessment of food handlers identified a lack of skills and knowledge around food safety and deficiencies in cleaning and sanitizing of food contact surfaces of equipment and utensils. The premises had no documented policy prohibiting food handlers from working while having a suspected foodborne illness. There was only one set of male and female toilets on the premises, with staff and guests using the same facilities.

\section{Public health intervention}

Three days following notification, the NSW Food Authority supervised a thorough cleaning of the premises and issued a prohibition order. No food preparation was undertaken by the business during the three days between notification of the incident and service of the prohibition order. This was the first time a prohibition order was issued in the absence of microbiological confirmation.

The order was issued on the basis that, given the number of ill attendees, lack of food safety skills and knowledge and potential for further infection of food handlers, there was a high risk of ongoing illness associated with the premises. The apparent inability to grasp the seriousness of an ill food handler working while infectious made non-compliance possible and the order necessary.

The prohibition order required the premise operators to undertake and provide details to the NSW Food Authority of measures to ensure all food handlers working were not having symptoms of foodborne illness, that all food handlers had skills and knowledge of food safety and hygiene appropriate to their work activities as per the Food Standards Code and that all utensils and surfaces were adequately cleaned and sanitized. ${ }^{14}$ The operators were also required to provide a list of staff cleared to work in food handling. Any person excluded from work was allowed to return only after being examined by a medical practitioner and cleared of any gastrointestinal illness.

Under the prohibition order, the business remained closed for two weeks while staff training was completed, the premises sanitized and re-inspected and documentation provided to show food handlers were no longer ill and were aware of health and hygiene requirements. Following this, a Certificate of Clearance was issued.

\section{Microbiological Investigation}

Stool samples were collected from eight guests and one food handler. Three stool specimens were positive for norovirus, five stool specimens (including the specimen from the food handler) were negative.

Four of the 22 environmental swabs - from the metal handle of a ladle from the kitchen, a tap in the ladies toilet, a microwave metal door and an oven handle - were positive for norovirus. Only one environmental swab (oven door handle) and stool samples from two 
guests could be genotyped, with all samples identified as a norovirus GIl.4 variant and identical to each other.

\section{DISCUSSION}

This investigation confirmed an outbreak of norovirus, with a possible cause being an infected food handler. The investigation highlights the role of timely and definitive regulatory action in minimizing public health risk where infection control breaches are suspected based on preliminary information, even before microbiological evidence is available.

Evidence of microbiological or chemical contamination and/or critical hygiene defects is usually required before a prohibition order can be issued. In this instance the initial outbreak investigation - and in particular indication of a sick food handler - provided sufficient evidence of ongoing risk to the public for the NSW Food Authority to issue a prohibition order while microbiological confirmation was being sought. As it was unknown if secondary transmission to other food handlers had occurred, the prohibition order prevented these staff from working until well after the period of exposure to norovirus ( 14 days) and required the function premises to remain closed until breaches of food safety (i.e. food handling practices by staff) were rectified.

Testing of all food handlers was considered but not pursued. People can continue to shed norovirus after symptoms cease and a positive norovirus result does not necessarily indicate a person is still infectious. There is no evidence that infected food handlers should be excluded from the workplace for longer than 48 hours after cessation of symptoms. ${ }^{11}$ Training food handlers and ensuring standard infection control procedures are followed at all times was believed to be the best way of minimizing the risk of future outbreaks at the premises.

Norovirus was isolated in three stool specimens and in four environmental swabs; the symptom profile and incubation period for cases is consistent with norovirus. The variant GII.4 is predominant in norovirus outbreaks globally. ${ }^{3}$ This strain was first identified in NSW in 2009 and has been the dominant GIl.4 variant in the state since then (personal communication, Peter White, 24 April 2012). The cohort was elderly and norovirus infection is more frequent in adults over 65 years. $^{3}$ Symptoms reported by guests were slightly unusual as diarrhoea was the most common symptom, rather than nausea or vomiting.

The investigation indicated a possible source of infection was an ill food handler: there was a clear description of a foodhandler who became ill before the event and was ill while working. Also, food preparation areas were found to be positive for norovirus. This indicates the virus spread from the kitchen to the function room. Two stool samples from ill patrons were also positive for norovirus with the same genotype as the environmental swab.

Transmission between guests, or as a result of environmental contamination in areas shared by guests and food handlers, such as the toilets, is also possible given the high prevalence of norovirus in the community. ${ }^{11}$ Two guests at Function A reported onset of illness only seven and eight hours afterward, indicating they may have been infected but asymptomatic at the function. Similarly, a guest who attended both Function $A$ and Function $B$ developed symptoms three hours after Function $B$. These guests are unlikely to be the sole cause of the outbreak given the unclear role of asymptomatic infection in transmission of norovirus. ${ }^{3,11}$

This epidemiological investigation was limited due to a full guest list not being available and only a subset of the total number of guests attending being interviewed. This resulted in incomplete information about the symptoms and food histories of all guests who attended the two functions. Relative risks for food exposures could not be reliably calculated, so foodborne transmission cannot be excluded. Ascertainment bias may have been introduced by asking for referrals from guests who had been ill, leading to overrepresentation of ill patrons in the sample.

The investigation team was also not able to obtain a full list of food handlers working over the weekend. Of the eight people for whom contact details were obtained, two refused to be interviewed or give personal information and one person could not be contacted. This may have resulted in under-ascertainment of cases among food handlers.

Norovirus was not detected in five specimens, including the specimen from the ill food handler despite symptoms consistent with norovirus infection. There are two potential reasons for the failure to detect norovirus 
in the specimens. One, the reported sensitivity and specificity of the RIDASCREEN norovirus kit varies widely from $71 \%-80.3 \%$ to $47 \%-100 \%$ respectively. ${ }^{15,16}$ The second reason relates to the quality and timeliness of stool samples. Samples must be collected as soon as possible after the onset of symptoms and ideally within three days for optimal results for testing with the RIDASCREEN norovirus EIA kit. The fact that the specimen from the food handler was collected at least one week after the onset of symptoms may have contributed to the negative result. ${ }^{17}$

This investigation highlights the importance of maintaining infection control in premises where food is served. Regular hand washing and cleaning and disinfection of premises should be promoted and food handlers exempted from work while they have symptoms of gastroenteritis. In cases with a demonstrable ongoing risk to public health, prohibition orders are effective measures in preventing further outbreaks while the premise is instituting infection control measures.

\section{Conflicts of interest}

None declared.

\section{Funding}

None.

\section{Acknowledgements}

Praveena Gunaratnam is employed as a trainee on the NSW Public Health Officer Training Programme, funded by the NSW Ministry of Health. She undertook this work based at the Sydney South West Public Health Unit. The Programme is offered in partnership with the University of NSW.

Typing of norovirus samples was undertaken by the Peter White Molecular Microbiology Laboratory, School of Biotechnology and Biomolecular Sciences, University of NSW. The authors of this paper wish to acknowledge helpful advice provided by Peter White and John-Sebastian Eden in interpretation of the results.

\section{References:}

1. Hall G et al.; OzFoodNet Working Group. Estimating foodborne gastroenteritis, Australia. Emerging Infectious Diseases, 2005, 11:1257-1264. doi:10.3201/eid1108.041367 pmid: 16102316
2. Liu B et al. An outbreak of Norwalk-like virus gastroenteritis in an aged-care residential hostel. New South Wales Public Health Bulletin, 2003, 14:105-109. doi:10.1071/NB03031 pmid:12907999

3. Division of Viral Diseases, National Center for Immunization and Respiratory Diseases, Centers for Disease Control and Prevention. Updated norovirus outbreak management and disease prevention guidelines. MMWR. Recommendations and Reports, 2011, 60 RR-3:1-18. pmid:21368741

4. Teunis PF et al. Norwalk virus: how infectious is it? Journal of Medical Virology, 2008, 80:1468-1476. doi:10.1002/ jmv.21237 pmid:18551613

5. Vivancos $\mathrm{R}$ et al. Food-related norovirus outbreak among people attending two barbeques: epidemiological, virological, and environmental investigation. International Journal of Infectious Diseases, 2009, 13:629-635. doi:10.1016/j.ijid.2008.09.023 pmid: 19147386

6. $\mathrm{Yu} \mathrm{JH}$ et al. Epidemiology of foodborne Norovirus outbreak in Incheon, Korea. Journal of Korean Medical Science, 2010, 25:1128-1133. doi:10.3346/jkms.2010.25.8.1128 pmid:20676321

7. Barrabeig $\mid \mathrm{I}$ et al. Foodborne norovirus outbreak: the role of an asymptomatic food handler. BMC Infectious Diseases, 2010, 10:269. doi:10.1186/1471-2334-10-269 pmid:20843351

8. Telfer B et al. A large outbreak of norovirus gastroenteritis linked to a catering company, New South Wales, October 2003. New South Wales Public Health Bulletin, 2004, 15:168-171. doi:10.1071/NB04036 pmid:15657625

9. Bresee JS et al. Foodborne viral gastroenteritis: challenges and opportunities. Clinical Infectious Diseases, 2002, 35: 748-753. doi: $10.1086 / 342386$ pmid: 12203173

10. Rockx B et al. Natural history of human calicivirus infection: a prospective cohort study. Clinical Infectious Diseases, 2002 35:246-253. doi:10.1086/341408 pmid:12115089

11. Guidelines for the public management of gastroenteritis outbreaks due to norovirus or suspected viral agents. Australian Government Department of Health and Ageing and Communicable Diseases Network Australia, 2010

12. Communicable Diseases Branch. Notifiable Diseases Manual. Sydney, New South Wales Health, 2008.

13. Food Act 2003 No 43, Section 60. Sydney, New South Wales, 2003.

14. Australia New Zealand Food Standards Code, Standard 3.2.2 Australian Government, 2006 (http://www.comlaw.gov.au/Details/ F2011C00591, accessed 6 October 2011).

15. Dimitriadis A, Marshall JA. Evaluation of a commercial enzyme immunoassay for detection of norovirus in outbreak specimens. European Journal of Clinical Microbiology and Infectious Diseases, 2005, 24:615-618. doi:10.1007/ s10096-005-0012-z pmid:16180034

16. Castriciano $\mathrm{S}$ et al. Comparison of the RIDASCREEN norovirus enzyme immunoassay to IDEIA NLV GI/GII by testing stools also assayed by RT-PCR and electron microscopy. Journal of Virological Methods, 2007, 141:216-219. doi:10.1016/j.jviromet.2006.12.001 pmid:17208311

17. RIDASCREEN Norovirus 3rd Generation, Article No. C 1401. R-Biopharm: Darmstadt, Germany. R-Biopharm, 2011 (http:// www.r-biopharm.com/product site. php?product id=33\&product class_one $=Q W 50$ aWdlbiBEZXRIY3Rpb24 $=$ \&product class two $=$ VmlydXNIcw $==\&$ product_class_three $=$ \&product_class four $=\&$ product range $=$ Food $\% 20$ and $\% 20$ Feed $\% 20$ Analysis $\&$, accessed 27 September 2011). 